\title{
Evaluación de la tolerancia al cobre de dos poblaciones de Oenothera picensis Phil. subsp. picensis (Onagraceae)
}

\section{Assessment of copper tolerance of two populations of Oenothera picensis Phil. subsp. picensis (Onagraceae)}

\author{
Isabel González $Z^{1} \&$ Alexander Neaman²* \\ ${ }^{1}$ Centro Regional de Innovación Hortofrutícola de Valparaíso-CERES, Pontificia Universidad Católica de Valparaíso, Chile. \\ ${ }^{2}$ Área del Medio Ambiente, Escuela de Agronomía, Pontificia Universidad Católica de Valparaíso, Quillota, Chile. \\ *alexander.neaman@ucv.cl
}

\begin{abstract}
RESUMEN
Oenothera picensis Phil. subsp. picensis puede crecer en suelos contaminados por cobre proveniente de una fundición de metal en Chile central, y presenta características que la hacen candidata para procesos de fitorremediación. Para conocer la tolerancia real y capacidad de extracción de cobre, y si ello ocurre sólo en la población expuesta, se realizaron ensayos hidropónicos utilizando dos poblaciones de $O$. picensis, provenientes de los sectores Los Maitenes y Maitencillo, áreas con concentraciones altas y normales de $\mathrm{Cu}$ en el suelo, respectivamente. Las plantas fueron expuestas a concentraciones de cobre entre 0 y $0,16 \mathrm{mM}$ en la solución, por un período de 1 mes. Las plantas mostraron síntomas de toxicidad a una concentración de $0,04 \mathrm{mM}$ de $\mathrm{Cu}$ en la solución, sin diferencias significativas en la tolerancia de ambas poblaciones. Las concentraciones de cobre foliar observadas en $O$. picensis alcanzaron un máximo de $1660 \pm 857 \mathrm{mg} \mathrm{kg}^{-1}$, en el tratamiento de $0,16 \mathrm{mM}$ de $\mathrm{Cu}$ en la solución. Sin embargo, en este tratamiento, la planta presentó severos síntomas de toxicidad. Por lo tanto, no es posible clasificarla como una especie hiperacumuladora.
\end{abstract}

Palabras clave: Hidroponía, toxicidad, fundición de cobre, parámetros de crecimiento, especie sensible.

\begin{abstract}
Oenothera picensis Phil. subsp. picensis can grow in soils that have been contaminated with copper from the metal smelting industry in central Chile, presenting characteristics that make it a candidate for phytoremediation processes. In order to know the real tolerance and capacity for copper extraction and to identify whether this characteristic is present solely in the exposed population, a hydroponic test was carried out using two populations of $O$. picensis, originated from Los Maitenes and Maitencillo, areas with high and normal $\mathrm{Cu}$ concentrations in the soil, respectively. The plants were exposed to copper concentration in solution between 0 and $0.16 \mathrm{mM}$, for one month. The plants presented symptoms of toxicity at $0.04 \mathrm{mM}$ of $\mathrm{Cu}$ in the solution, without significant differences in the tolerance of both populations. The shoot copper concentrations observed in $O$. picensis reached the maximum of $1660 \pm 857 \mathrm{mg} \mathrm{kg}^{-1}$, in the treatment of $0.16 \mathrm{mM}$ of Cu in the solution. However, in this treatment, the plant presented severe toxicity symptoms. Thus, it is not possible to classify it as a hyperaccumulating species.
\end{abstract}

KeYwords: Hydroponic, toxicity, copper smelter, growth parameters, sensitive specie.

\section{INTRODUCCIÓN}

Los suelos del Valle de Puchuncaví, en la zona costera de Chile central, han sido expuestos a las emanaciones provenientes del Complejo Industrial Ventanas desde 1964 (Folchi 2006). Actualmente, en los sitios aledaños al complejo industrial, los suelos son ácidos, están severamente erosionados y contienen altas concentraciones de metales (cobre, zinc, plomo y cadmio) y metaloides (arsénico)
(González \& Berqvist 1986a, González \& Ite 1992, Ginocchio et al. 2004). Estos sitios presentan baja riqueza de especies, cobertura vegetal evidentemente disminuida y pobre banco de semillas, en comparación con sitios no contaminados del Valle de Puchuncaví (Ginocchio 2000).

En los sitios ácidos y metalíferos, solamente son capaces de sobrevivir y reproducirse aquellas especies y poblaciones vegetales que presentan resistencia a las condiciones adversas del suelo (MacNair 1993, Whiting et al. 2002). Es probable 
por lo tanto, que dentro de la diversidad observada en sitios metalíferos, algunas de estas especies pertenezcan al grupo de las metalófitas, es decir resisten la toxicidad mediante diversos mecanismos celulares (Whiting et al. 2004). Existen tres categorías dentro de este grupo de plantas, las metalófitas obligadas, endémicas de los sitios metalíferos (generalmente vetas de mineral) con una distribución geográfica restringida (Baker \& Brooks 1989); las "metalófitas facultativas" desarrolladas sólo en presencia de un metal específico en el suelo, y las pseudometalófitas, cuya tolerancia no depende de la presencia del metal (Baker et al. 2010).

La fitorremediación es una técnica que utiliza el cultivo de plantas metalófitas in situ para remover los metales del suelo (fitoextracción) o estabilizarlos en éste (fitoestabilización). González et al. (2008) realizaron una prospección de especies potencialmente metalófitas para ser utilizadas en procesos de fitorremediación de cobre en el sector Los Maitenes, $3 \mathrm{~km}$ al oriente del Complejo Industrial Ventanas, localidad identificada como una de las más afectadas por la actividad del Complejo Industrial (Bruno 1992, De Gregori et al. 2000). La prospección permitió identificar 22 especies, entre ellas destacó Oenothera picensis Phil. subsp. picensis (tratada como Oenothera affinis por González et al. 2008; en adelante referida como Oenothera picensis) por la mayor concentración de cobre acumulado en su biomasa aérea, con un promedio de $614 \pm$ $4,6 \mathrm{mg} \mathrm{kg}^{-1}$. La especie es una hierba perenne, nativa, con alta producción de biomasa, resistente a la sequía y muy fácil de propagar (Matthei 1995); debido a estas características, tiene potencial para ser usada en procesos a gran escala de fitoextracción de cobre desde el suelo. Para comprobar la real tolerancia y capacidad de acumulación de cobre en condiciones controladas, los ensayos hidropónicos surgen como una herramienta eficiente debido a que permiten un control efectivo de la disponibilidad de metales, además de resultados rápidos y precisos (Giri \& Patel 2012).

El objetivo del presente trabajo fue evaluar la tolerancia al cobre y la capacidad de acumulación de $\mathrm{Cu}$ en dos poblaciones de Oenothera picensis, la primera situada en un sitio expuesto históricamente a contaminación cúprica del Complejo Industrial Ventanas y otra proveniente de un sitio no expuesto a dicha contaminación. Además, se determinaron los límites de tolerancia y acumulación de cobre en tejidos aéreos, para evaluar la utilidad de la especie para procesos de fitoextracción.

\section{MATERIALES Y MÉTODOS}

Material vegetal

Se obtuvieron semillas de Oenothera picensis en dos sitios del valle de Puchuncaví: (1) Los Maitenes (32 51'54,7" S; $71^{\circ} 25^{\prime} 46,2^{\prime \prime}$ O) a $2 \mathrm{~km}$ al oriente del Complejo Industrial Ventanas, un sitio altamente contaminado por cobre (González \& Berqvist 1986b, Bruno 1992, De Gregori et al. 2000) y (2) Maitencillo ( $32^{\circ} 40^{\prime} 27,1^{\prime \prime} \mathrm{S} ; 7^{\circ} 25^{\prime} 35,8^{\prime \prime} \mathrm{O}$ ), a $11 \mathrm{~km}$ al norte del Complejo Industrial, no afectado por contaminación cúprica (Muena et al. 2010). Se recolectaron semillas de 5 individuos por sitio, las cuales fueron homogeneizadas.

Las semillas de ambas poblaciones fueron germinadas en un sustrato de perlita/mulch 1:1 en bandejas de poliestireno, bajo iluminación natural a través de ventanales de vidrio de exposición norte, con temperaturas máxima/mínima de $7 / 24{ }^{\circ} \mathrm{C}$ y una radiación fotosintéticamente activa con un máximo de $1.100 \mu \mathrm{mol} \mathrm{m} \mathrm{m}^{-2} \mathrm{~s}^{-1}$.

CARACTERIZACión de Los SUELOS DE ORIGeN

Para comprobar la diferencia en la contaminación cúprica de cada sitio, se realizó una caracterización de suelos (Tabla I), tomando 10 muestras de aproximadamente 0,6 $\mathrm{kg}$ compuestas de 3 submuestras de 0,2 $\mathrm{kg}$, en cada uno de los sitios de los cuales se recolectaron las semillas, en transectos de aproximadamente $1 \mathrm{~km}$ en Maitencillo y Los Maitenes. Se determinó la concentración total de $\mathrm{Cu}$ mediante espectrofotometría de absorción atómica (GBC, model 902) luego de la digestión del suelo con ácidos nítrico y perclórico (Verlinden 1982). Para el aseguramiento de calidad se utilizó una muestra de referencia certificada (GRX-2) obtenida del United States Geological Survey.

TABLA I. Caracterización físico-química de los suelos superficiales donde fueron recolectadas las poblaciones estudiadas de Oenothera picensis.

TABLE I. Physico-chemical characteristics of the topsoils where studied populations of Oenothera picensis were collected.

\begin{tabular}{cccc}
\hline VARIABLE & UNIDAD & Los MAITENES & MAITENCILLO \\
\hline Cu total & $\mathrm{mg} \mathrm{kg}^{-1}$ & $652 \pm 120$ & $85 \pm 13$ \\
$\mathrm{Cu}$ soluble & $\mathrm{mg} \mathrm{kg}^{-1}$ & $27 \pm 18$ & $0,01 \pm 0,04$ \\
$\mathrm{pH}$ & & $4,7 \pm 0,3$ & $6,3 \pm 0,2$ \\
$\mathrm{pCu}^{2+}$ & & $4,7 \pm 0,9$ & $8,0 \pm 0,6$ \\
Materia orgánica & $\%$ & $2,3 \pm 0,1$ & $2,1 \pm 0,4$ \\
Textura & & Arenoso franca & Arenosa \\
\hline
\end{tabular}


Las concentraciones de $\mathrm{Cu}$ soluble, $\mathrm{pCu}^{+2}$ y $\mathrm{pH}$ se determinaron en soluciones de $\mathrm{KNO}_{3}$ (relación 1:2,5 suelo/ agua) (Stuckey et al. 2009). El valor de $\mathrm{pCu}^{+2}\left(\mathrm{pCu}^{+2}=\right.$ $-\log \left[\mathrm{Cu}^{+2}\right]$, donde $\left[\mathrm{Cu}^{+2}\right]$ es la actividad del ión libre $\mathrm{Cu}^{+2}$ ) se determinó mediante un electrodo selectivo del ión $\mathrm{Cu}^{+2}$ (Orion 96-29 ionplus). El electrodo fue calibrado usando soluciones tampón de ácido iminodiacético (Rachou et al. 2007). La concentración de $\mathrm{Cu}$ soluble se determinó por espectrofotometría de absorción atómica.

\section{ENSAYO HIDROPÓNICO}

Se realizó un ensayo hidropónico sometiendo las plantas de las dos poblaciones de Oenothera picensis a crecientes concentraciones de cobre. Para los ensayos se utilizó agua potable por los grandes volúmenes requeridos, que superaban la capacidad del equipo de osmosis. Una vez que las plántulas de $O$. picensis desarrollaron 4 hojas, fueron lavadas cuidadosamente con agua potable para remover las partículas del sustrato y transferidas a recipientes plásticos de $6 \mathrm{~L}$ que contenían solución Hoagland modificada para evitar sobresaturación y precipitación del $\mathrm{Cu}$. Para esto, en primer lugar, se determinaron las concentraciones macronutrientes y micronutrientes en agua potable según los métodos de Sadsawka (2006), posteriormente se agregaron soluciones estándares de iones en cantidad tal para alcanzar las concentraciones indicadas en la solución Hoagland tradicional. En segundo lugar, cada vez que se cambió la solución nutritiva, se adicionó $\mathrm{HNO}_{3} 0,6 \mathrm{M}$ a cada recipiente, para ajustar el $\mathrm{pH}$ a 5,0. La solución Hoagland se cambió en cada recipiente cada 3 días y se mantuvo aireada continuamente utilizando una bomba de aire para acuario (con un flujo de aire de $1,8 \mathrm{~m}^{3} \mathrm{~h}^{-1}$ ) y difusores de aire.

Luego del ajuste del $\mathrm{pH}$, las concentraciones finales de iones en los recipientes fueron: $\mathrm{K}^{+}$2,13 $\mathrm{mM}^{2} \mathrm{NO}_{3}{ }^{-2}$, 4 $\mathrm{mM} ; \mathrm{NH}_{4}^{+} 0,88 \mathrm{mM} ; \mathrm{PO}_{4}^{-3} 0,4 \mathrm{mM} ; \mathrm{Na}^{+} 1,06 \mathrm{mM} ; \mathrm{Ca}^{+2} 0,49$ $\mathrm{mM} ; \mathrm{Mg}^{+2} 0,36 \mathrm{mM} ; \mathrm{SO}_{4}^{-2} 0,4 \mathrm{mM} ; \mathrm{Fe}^{+2} 0,031 \mathrm{mM} ; \mathrm{Mn}^{+2}$ 0,0033 mM; $\mathrm{BO}_{3}^{-3} 0,012 \mathrm{mM} ; \mathrm{Zn}^{+2} 0,002 \mathrm{mM} ; \mathrm{Cu}^{+2} 0,0013$ $\mathrm{mM} ; \mathrm{MoO}_{4}^{-2}$ 0,0002 mM. Según Kopittke et al. (2010), la presencia de al menos $\mathrm{Ca}$ y $\mathrm{B}$ en la solución es necesaria para la legitimidad de los ensayos hidropónicos.

Luego de una semana de adaptación, se aplicaron diferentes concentraciones de solución madre de $\mathrm{CuSO}_{4}$ (pentahidratado) $1 \mathrm{M}$. En este ensayo se utilizaron 5 niveles de concentración: $0 ; 0,04 ; 0,08 ; 0,12 ; 0,16 \mathrm{mM}$ de $\mathrm{Cu}$, agregando 0,$24 ; 0,48 ; 0,72$ y $0,96 \mathrm{~mL}$ de la solución madre a los recipientes de $6 \mathrm{~L}$, respectivamente. Estas concentraciones de $\mathrm{Cu}$ corresponden a valores de $\mathrm{pCu}^{+2}$ de 6,$1 ; 4,6 ; 4,3 ; 4,1$ y 4,0, respectivamente (calculado por Visual MINTEQ 3.0).

Una vez transcurridas $48 \mathrm{~h}$ desde la aplicación de los tratamientos, se tomaron muestras $(25 \mathrm{~mL})$ en los recipientes con la mayor concentración de cobre, se filtraron utilizando papel filtro $\mathrm{N}^{\circ} 2$ y se determinó las concentraciones de $\mathrm{Cu}$, $\mathrm{P}, \mathrm{Fe}$ y $\mathrm{Ca}$ en la solución según los métodos de Sadsawka
(2006), asegurando así que la solución no estuviera sobresaturada.

El sistema hidropónico consistió de tres recipientes para cada concentración de cobre. En cada recipiente se dispusieron tres individuos de cada población, soportados en planchas de poliestireno. Los recipientes, dispuestos al azar, fueron cambiados de posición una vez a la semana, para eliminar el efecto de las variaciones de luz.

El ensayo se realizó durante el mes de junio de 2011, con un fotoperíodo de $10 \mathrm{~h}$ y una temperatura mínima/máxima de $17 / 25^{\circ} \mathrm{C}$, desarrollándose en una sala con iluminación natural a través de ventanales de vidrio con exposición norte con un máximo de radiación fotosintéticamente activa con un máximo de $1100 \mu \mathrm{mol} \mathrm{m} \mathrm{m}^{-2} \mathrm{~s}^{-1}$. La duración del ensayo fue de un mes. Antes de desmontar el ensayo, en cada hoja se determinó el porcentaje de su superficie con clorosis $(0,25$, 50,75 y $100 \%$ ), en base a un examen visual.

\section{ANÁlisis DE TEJIDOS}

Al finalizar el ensayo se determinó la concentración de cobre en los tejidos aéreos y en raíces de las plantas, así como la biomasa de estos tejidos, separando los tejidos aéreos de las raíces. El material vegetal fue cuidadosamente lavado en el siguiente orden: agua potable, $\mathrm{HCl} \mathrm{0,01} \mathrm{M,} \mathrm{agua}$ desionizada, EDTA 0,05 M, agua desionizada (Steubing 1982). El material vegetal fue secado en una estufa a $60^{\circ} \mathrm{C}$ por $48 \mathrm{~h}$ y triturado en un molino de acero inoxidable (IKA, modelo A11 Basic), previa determinación de su biomasa. El material vegetal fue calcinado en una mufla (Vulcan, modelo A-550) a $500{ }^{\circ} \mathrm{C}$ para posteriormente agregar ácido clorhídrico, calentar en placa hasta ebullición, enfriar y filtrar (Sadzawka et al. 2007). El cobre en la solución obtenida fue determinado mediante espectrofotometría de absorción atómica. En el caso de que un individuo no presentara suficiente biomasa para ser analizado independientemente, se hizo una muestra compuesta con los individuos de un mismo recipiente.

Para comprobar la exactitud y precisión del método, se llevaron a cabo análisis de dos muestras de referencia: 1570a (espinaca) y 1573a (tomate) del National Institute of Standards and Technology. La diferencia entre los valores obtenidos y los de referencia fue menor al 5\% en ambas muestras.

\section{ANÁlisis DE DATOS}

Al concluir el ensayo se realizaron los siguientes cálculos: (1) La relación concentración de $\mathrm{Cu}$ en tejidos aéreos/ concentración de $\mathrm{Cu}$ en raíz (en adelante $\mathrm{Cu}_{\mathrm{ta}} / \mathrm{Cu}_{\mathrm{r}}$ ). Este valor permite evaluar la tasa de translocación de cobre desde la raíz hacia los tejidos aéreos y con ello vislumbrar si la tolerancia se basa en la exclusión o en la acumulación. (2) Extracción total de cobre por la planta, se calculó multiplicando la concentración de cobre en los tejidos aéreos por la biomasa aérea producida, en cada individuo. 
Se aplicó análisis ANDEVA (Análisis de Varianza) y prueba de Tukey al 95\% de confianza para determinar diferencias significativas entre las poblaciones y entre los tratamientos (ANDEVA de dos vías) con respecto a las variables respuesta: porcentaje de clorosis, biomasa aérea, biomasa radical, concentración de cobre en tejidos aéreos, concentración de cobre en raíces y extracción total de cobre por la planta. Se utilizó el programa Software Minitab 15 para Windows.

\section{RESULTADOS}

EFectos de LAS CONCENTRACIONES DE COBRE EN LA SOLUCiÓN SOBRE EL DESARROLLO DE LA PLANTA

La concentración de cobre en la solución tuvo efecto significativo sobre el porcentaje de clorosis, la biomasa aérea y la biomasa radical $(\mathrm{P}<0,001$ en los tres casos, Tabla II). La concentración menor de cobre en solución
$(0,04 \mathrm{mM})$ indujo clorosis y disminuyó significativamente la biomasa aérea y radical, en relación al control (Fig. 1 A, B y C). Sin embargo, a mayores concentraciones de cobre en la solución, no hubo diferencias significativas entre las concentraciones respecto a estas tres variables respuesta. Tampoco hubo diferencias significativas entre las poblaciones de ambos sitios respecto al porcentaje de clorosis y la biomasa aérea o radical (Tabla II).

CONCENTRACIÓN DE COBRE EN TEJIDOS AÉREOS Y EN RAíCES La concentración de cobre en la solución afectó significativamente la concentración de cobre en los tejidos aéreos y en las raíces $(\mathrm{P}<0,001$, Tabla II). A mayor concentración de cobre en la solución, se observó una mayor concentración de cobre en los tejidos aéreos y en las raíces, en ambas poblaciones (Fig. 1D y 1E). No hubo diferencias entre las poblaciones respecto de estas variables (Tabla II). Frente a la mayor concentración de cobre en la solución evaluada $(0,16 \mathrm{mM})$, se determinó una concentración de

TABLA II. ANDEVA para las variables clorosis, biomasa, concentración de cobre en tejidos aéreos y raíces de Oenothera picensis, y extracción total de $\mathrm{Cu}$ por la especie.

TABLE II. ANOVA for variables of chlorosis, biomass, copper concentration in aboveground tissues and roots of Oenothera picensis, and total $\mathrm{Cu}$ extraction by the species.

\begin{tabular}{|c|c|c|c|}
\hline & GRADOS DE LIBERTAD & $\mathrm{F}$ & Probabilidad (p) \\
\hline \multicolumn{4}{|c|}{ Clorosis } \\
\hline Concentración de $\mathrm{Cu}(\mathrm{A})$ & 4 & 17,93 & $<0,05$ \\
\hline Origen plántulas (B) & 1 & 2,83 & 0,96 \\
\hline$A^{*} B$ & 4 & 0,16 & 0,10 \\
\hline \multicolumn{4}{|c|}{ Biomasa aérea } \\
\hline Concentración de $\mathrm{Cu}(\mathrm{A})$ & 4 & 17,53 & $<0,05$ \\
\hline Origen plántulas (B) & 1 & 0,67 & 0,41 \\
\hline$A^{*} B$ & 4 & 0,70 & 0,59 \\
\hline \multicolumn{4}{|c|}{ Biomasa radical } \\
\hline Concentración de $\mathrm{Cu}(\mathrm{A})$ & 4 & 23,64 & $<0,05$ \\
\hline Origen plántulas (B) & 1 & 0,21 & 0,65 \\
\hline$A * B$ & 4 & 0,05 & 0,97 \\
\hline \multicolumn{4}{|c|}{ Concentración de $\mathrm{Cu}$ en tejidos aéreos } \\
\hline Concentración de $\mathrm{Cu}(\mathrm{A})$ & 4 & 9,02 & $<0,05$ \\
\hline Origen plántulas (B) & 1 & 1,17 & 0,29 \\
\hline $\mathrm{A} * \mathrm{~B}$ & 4 & 2,57 & 0,06 \\
\hline \multicolumn{4}{|c|}{ Concentración de $\mathrm{Cu}$ en raíces } \\
\hline Concentración de $\mathrm{Cu}(\mathrm{A})$ & 4 & 62,99 & $<0,05$ \\
\hline Origen plántulas (B) & 1 & 2,97 & 0,1 \\
\hline$A * B$ & 4 & 0,84 & 0,51 \\
\hline \multicolumn{4}{|c|}{ Extracción total } \\
\hline Concentración de $\mathrm{Cu}(\mathrm{A})$ & 4 & 5,19 & $<0,05$ \\
\hline Origen plántulas (B) & 1 & 0,73 & 0,40 \\
\hline $\mathrm{A}^{*} \mathrm{~B}$ & 4 & 1,48 & 0,23 \\
\hline
\end{tabular}


cobre en los tejidos aéreos de $1660 \pm 857 \mathrm{mg} \mathrm{kg}^{-1}$ y en raíces de $14.082 \pm 4.050 \mathrm{mg} \mathrm{kg}^{-1}$, sin embargo en las plantas se evidenció signos de toxicidad.

La relación $\mathrm{Cu}_{\mathrm{ta}} / \mathrm{Cu}_{\mathrm{r}}$ varió en el intervalo de 0,1 a 0,14 , siendo estadísticamente similar entre las poblaciones provenientes de ambos sitios y entre las concentraciones de cobre en la solución ( $\mathrm{P}>0,05$, datos no mostrados).

\section{EXTRACCIÓN TOTAL DE COBRE}

La concentración de cobre en la solución afectó significativamente la extracción total de $\mathrm{Cu}(\mathrm{P}<0,005$, Tabla II). A mayor concentración de cobre en la solución, se observó una mayor extracción total de cobre por las plantas (Fig. 1F). No hubo diferencias significativas $(\mathrm{P}>0,05)$ entre ambos sitios respecto de la extracción total de cobre por las plantas (Tabla II). Para la mayor concentración de cobre en la solución evaluada $(0,16 \mathrm{mM})$, la extracción total de cobre por $O$. picensis fue de $34 \pm 23 \mathrm{mg}_{\text {planta }}{ }^{-1}$.

\section{DISCUSIÓN}

\section{ENSAYOS HIDRÓPÓNICOS}

El ajuste de pH a la solución Hoagland se realizó para evitar la precipitación de compuestos que ocurría con el pH neutro de la solución, principalmente al adicionar las soluciones de $\mathrm{CuSO}_{4} \cdot \mathrm{Al}_{\text {observar esta situación, se realizó el cálculo de los }}$ índices de saturación de compuestos, utilizando el software Visual MINTEQ 3.0 (Gustafsson 2013). El software demostró que el $\mathrm{pH}$ de la solución debe ser $\leq 5,0$ para evitar la sobresaturación de la solución respecto a los compuestos de $\mathrm{Cu}, \mathrm{Fe}, \mathrm{Mn}$ y P. El pH 5,0 es similar al reportado en los suelos de Los Maitenes (Muena et al. 2010, Goecke et al. 2011), asemejándose al escenario real para el desarrollo de la plantas. Según Kopittke et al. (2010), el control del pH en la solución, tal como se realizó en el presente ensayo, se considera un factor relevante para la correcta interpretación de los resultados de los ensayos hidropónicos.

En relación a los niveles de concentraciones de $\mathrm{Cu}$ utilizadas, Kopittke et al. (2010), indica que un ensayo hidropónico debe contener al menos 4 concentraciones del metal a evaluar, incluyendo el testigo, por lo que se considera que el número (5) de niveles utilizado en este ensayo fue adecuado. Los valores máximos y mínimos de concentraciones utilizados para los ensayos hidropónicos se establecieron tomando como base investigaciones internacionales (Tabla III), pero considerando que en la investigación se postula que Oenothera picensis es una especie tolerante. Por esto, se usaron concentraciones relativamente altas y levemente mayores a las encontradas en terreno, con el objeto de testear la tolerancia máxima de la especie. El rango de $\mathrm{pCu}^{+2}$ encontrado en terreno va de 5 a 9 , mientras que el rango utilizado en este ensayo va de 4 a 6 (el valor de $\mathrm{pCu}^{+2}$ es inversamente proporcional a la actividad del ion $\mathrm{Cu}^{+2}$ en la solución).

Respecto a los intervalos de concentraciones de $\mathrm{Cu}$ utilizadas, un análisis de las concentraciones de Cu utilizadas por varios autores (Tabla III) permitió concluir que no existe un consenso respecto al intervalo de concentraciones de cobre que se debe utilizar en ensayos hidropónicos, existiendo una alta variabilidad. El intervalo $(0,04 \mathrm{mM})$ utilizado entre las concentraciones se estableció en base a investigaciones en otras especies (Ali et al. 2002, Borghi et al. 2007).

La duración del ensayo se decidió considerando que el objetivo fue determinar la tolerancia al cobre de la especie y su máxima capacidad de acumulación. Si bien la tolerancia puede determinarse en pocos días (7-12 días) evaluando los síntomas de toxicidad, la evaluación de la acumulación requiere más tiempo. Al respecto, Ebbs \& Kochian (1998), Kuzovkina et al. (2004) y Lou et al. (2004) realizaron ensayos hidropónicos de aproximadamente un mes, para determinar la tolerancia al $\mathrm{Cu}, \mathrm{Cd}$ y/o $\mathrm{Zn}$ y extracción de estos metales por diferentes especies vegetales (Tabla III). Asimismo los autores (González et al. 2008) identifican el fin del estado vegetativo como el punto de mayor extracción de $\mathrm{Cu}$.

CARACTERísticAs de LOS SUELOS DE ORIGEN DE LAS POBLACIONES Las características químicas de los suelos de Los Maitenes, principalmente las concentraciones de cobre total y soluble, $\mathrm{pCu}^{+2}$ y $\mathrm{pH}$ (Tabla I), evidencian el impacto del complejo industrial. La concentración de cobre soluble es alta en comparación con otros suelos contaminados con cobre de la región $\left(0,47 \mathrm{mg} \mathrm{kg}^{-1}\right.$ en el valle de Catemu) (Ávila et al. 2007, Ávila et al. 2009) y con el suelo de Maitencillo (Tabla I). El valor de $\mathrm{pCu}^{+2}$ en el suelo de Los Maitenes es bajo, es decir, la actividad del ion $\mathrm{Cu}^{+2}$ es alta, en relación a otros lugares contaminados por cobre de la región (por ejemplo $\mathrm{pCu}^{+2}$ de 7,9 en el valle de Catemu, Ávila et al. 2007, Ávila et al. 2009) y al suelo de Maitencillo (Tabla I). El Cu $\mathrm{Cu}^{+2}$ corresponde a la fracción más tóxica de cobre en el suelo (Sauvé et al. 1997); por lo tanto, una alta actividad implica mayor toxicidad. En consecuencia, puede afirmarse que la disponibilidad de $\mathrm{Cu}$ en Los Maitenes es alta.

Para los fines de la presente investigación, los suelos de ambos sectores son comparables debido a que tienen textura y contenido similar de materia orgánica (Tabla I). Ambos suelos corresponden a paleodunas estabilizadas (Cosio et al. 2007) y fueron clasificados como Entisoles (Soil Survey Staff 2003).

Tolerancia AL COBRE DE LAS POBlaCiOnes DE OENOTHERA PICENSIS

La concentración de $\mathrm{Cu}^{+2} 0,04 \mathrm{mM}$ causó efectos negativos sobre las plantas de $O$. picensis (Tabla II). El análisis de diversos ensayos hidropónicos de tolerancia al cobre por plantas no ha permitido llegar a un consenso para 
determinar en qué circunstancias una especie o población es considerada tolerante al cobre o no. Sin embargo, en investigaciones previas (ver Tabla III) se indica que aquellas plantas consideradas tolerantes o indicadoras son capaces de soportar al menos concentraciones de 0,1 mM de $\mathrm{Cu}$. En consecuencia $O$. picensis corresponde a una especie sensible ya que a los 0,04 mM presentó síntomas de toxicidad. $\mathrm{Si}$ bien la relación $\mathrm{Cu}_{\mathrm{ta}} / \mathrm{Cu}_{\mathrm{r}}$ fue muy inferior a 1 en ambas poblaciones, lo que demuestra que la planta se comporta como excluyente (Chipeng et al. 2010), restringiendo la translocación desde las raíces a los tejidos aéreos, la especie no fue capaz de tolerar las altas concentraciones de cobre en el medio.

Según los resultados obtenidos y la definición de Baker et al. (2010), Oenothera picensis debería ser considerada como una especie "acompañante no tolerante". Estas especies, al estar en suelos metalíferos presentan poco vigor, son comúnmente anuales y persisten poco tiempo en el sitio (a largo plazo). Sin embargo, González et al. (2008) señalan que la población de $O$. picensis presente en Los Maitenes es al menos bianual —dado que se pudo observar vestigios de temporadas anteriores en los individuos - con una considerable producción de biomasa (30 a $170 \mathrm{~g}$ de masa seca en estado de inicio de floración) en relación a otras especies encontradas en el sitio, y ha sido registrada en el sitio desde el año 1997 (Ginocchio 1997).

Una probable explicación de este comportamiento podría deberse a que el cobre no es el principal factor limitante que afecta la composición de la comunidad vegetal en Los Maitenes. En efecto, Ginocchio (2000) indica que el nitrógeno presente en el suelo explica un $13 \%$ de la variación en la abundancia de las comunidades vegetales evaluadas a lo largo de un transecto de 5,5 $\mathrm{km}$ hacia el SSE desde el complejo industrial. Mientras que el pH del suelo explicaría un $10 \%$ de la variación en la abundancia y el $\mathrm{Cu}$ extractable sólo un 7\%.

Por otro lado, los sitios donde se obtuvieron las

TABLA III. Síntesis de resultados de ensayos hidropónicos que evalúan el efecto del cobre sobre el desarrollo de especies. Cu* = concentración de $\mathrm{Cu}$ en la solución a la cual se observan los primeros síntomas de toxicidad $(\mathrm{mM}), \mathrm{Cu}^{* *}=$ intervalo de concentraciones evaluadas en el ensayo (mM). ${ }^{1}$ Proveniente de sitio contaminado; ${ }^{2}$ Proveniente de sitio no contaminado; ${ }^{3}$ En comparación a otra población de la misma especie.

TABLE III. Summary of results of hydroponic tests that evaluate the effect of copper on the development of species. Cu* $=$ copper concentration in the solution that produces the first toxicity symptoms $(\mathrm{mM}), \mathrm{Cu}^{* *}=$ concentration range evaluated in the test (mM). ${ }^{1} \mathrm{From}$ a contaminated site; ${ }^{2}$ From a non-contaminated site; ${ }^{3}$ In comparison with other population of the same species.

\begin{tabular}{|c|c|c|c|c|c|c|}
\hline Autor & EsPeCIE & VARIABLE & $\mathrm{Cu}^{*}$ & $\mathrm{Cu}^{* *}$ & $\begin{array}{c}\text { DURACIÓN DEL } \\
\text { EXPERIMENTO } \\
\text { (DÍAS) }\end{array}$ & $\begin{array}{l}\text { CATEGORÍA DE } \\
\text { TOLERANCIA }\end{array}$ \\
\hline Lou et al. (2004) & Elsholtzia haichowensis & & 0,3 & $0,01-0,5$ & 6 & Tolerante \\
\hline Borghi et al. (2007) & $\begin{array}{l}\text { Populus } \times \text { euramericana clone } \\
\text { Adda }\end{array}$ & $\begin{array}{l}\text { Biomasa } \\
\text { radical }\end{array}$ & 0,1 & $0,004-0,1$ & $15-35$ & Tolerante \\
\hline Quian et al. (2005) & Elsholtzia haichowensis & & 0,1 & $0,0032-0,1$ & 14 & Tolerante \\
\hline Wenshan et al. (2007) & Rumex japonicus $^{1}$ & & 0,1 & $0,025-0,1$ & 14 & Tolerante $^{3}$ \\
\hline Borghi et al. (2007) & $\begin{array}{l}\text { Populus } \times \text { euramericana clone } \\
\text { Adda }\end{array}$ & & 0,1 & $0,025-0,075$ & 80 & Tolerante \\
\hline Lou et al. (2004) & Elsholtzia haichowensis & Biomasa aérea & 0,5 & $0,01-0,5$ & 6 & Tolerante \\
\hline Ali et al. (2002) & Phragmites australis & & 0,08 & $0,008-0,16$ & 15 & Tolerante \\
\hline Ali et al. (2002) & Zea mays & & 0,008 & $0,008-0,16$ & 15 & No tolerante \\
\hline Wenshan et al. (2007) & Rumex japonicus ${ }^{2}$ & & 0,025 & $0,025-0,1$ & 14 & No tolerante $^{3}$ \\
\hline Ali et al. (2002) & Phragmites australis & & $>0,16$ & $0,008-0,16$ & 15 & Tolerante \\
\hline Ali et al. (2002) & Zea mays & Clorosis & 0,08 & $0,008-0,16$ & 15 & No tolerante \\
\hline Schiavon et al. (2007) & $\begin{array}{l}\text { Arabidopsis thaliana } \\
\text { accesions Columbia }\end{array}$ & & 0,04 & $0,04-0,05$ & 14 & No tolerante \\
\hline
\end{tabular}


poblaciones de Oenothera picensis son zonas áridas, con un régimen histórico anual de precipitaciones de $300 \mathrm{~mm}$ y que ha disminuido significativamente en la última década, llegando a valores de 140-180 mm en las zonas costeras en los años 2011-2012 (SEREMI de Agricultura, 2013), con una disponibilidad real de metales en el suelo reducida $y$, por lo tanto, una baja exposición a los efectos tóxicos.

Además, se debe considerar que en condiciones reales
A

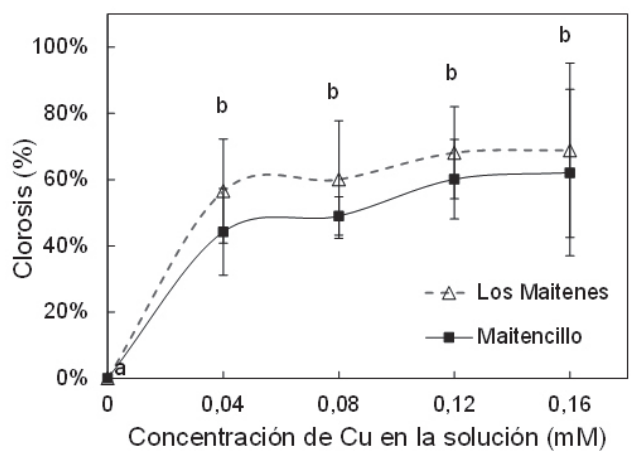

C

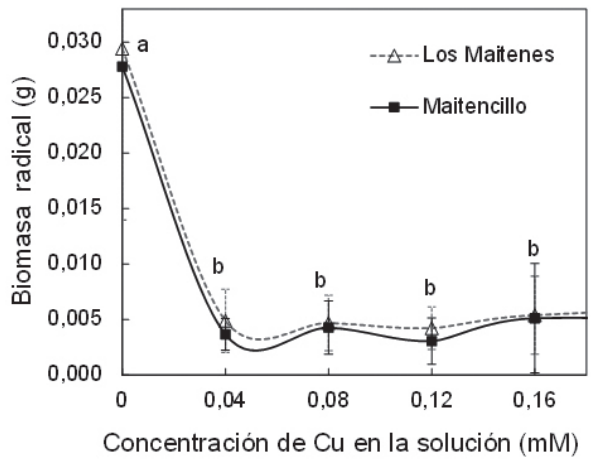

$\mathrm{E}$

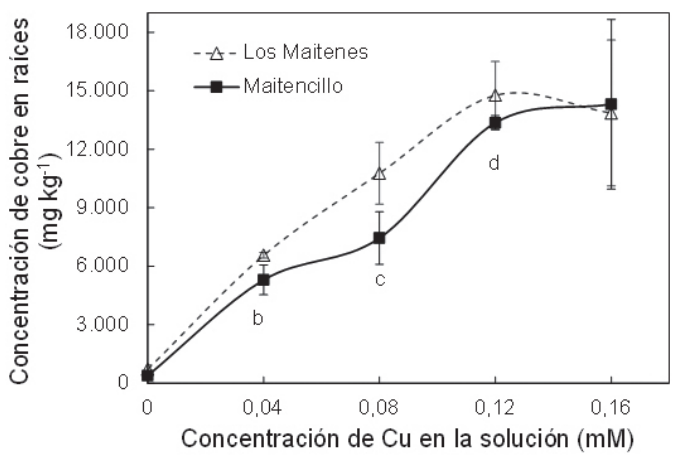

$\mathrm{B}$

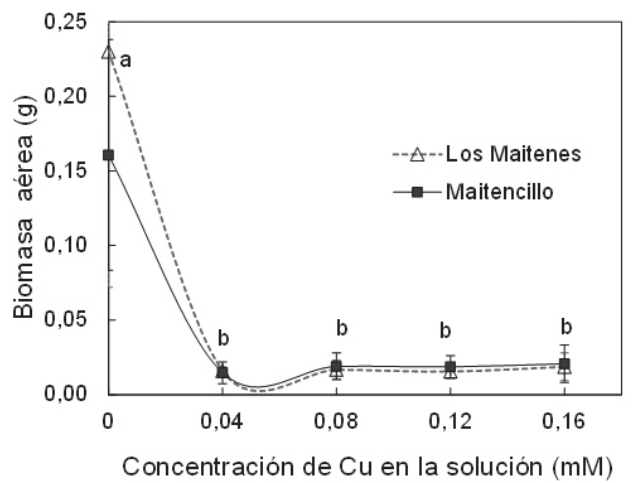

$\mathrm{D}$

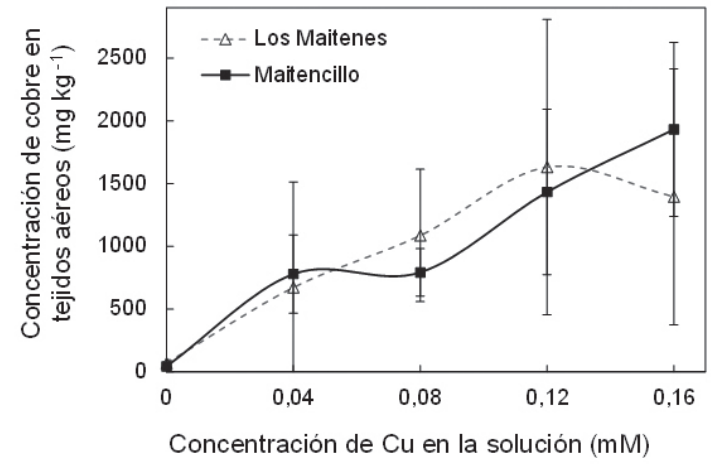

F

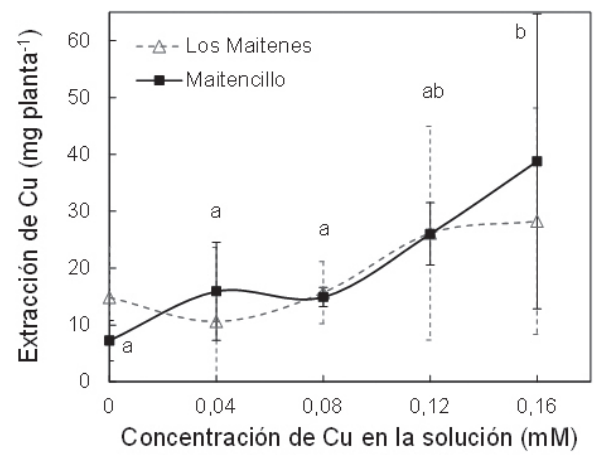

FiguRA 1. Efecto de la concentración de cobre en la solución sobre la clorosis (A), producción de biomasa aérea (B), producción de biomasa radical (C), concentración de cobre en tejidos aérea (D), concentración de cobre en raíces (E), y extracción total de cobre (F), en dos poblaciones de Oenothera picensis. Se indica la media y la desviación estándar para cada concentración de Cu. Letras diferentes indican diferencias significativas entre las distintas concentraciones de $\mathrm{Cu}$. No hubo diferencias significativas entre las poblaciones $(\mathrm{P}>0,05)$.

FigURE 1. Effect of copper concentration in the solution on the chlorosis (A), aboveground biomass production (B), root biomass production (C), copper concentration in aboveground biomass (D), copper concentration in roots (E) and total copper extraction (F), in two populations of Oenothera picensis. The mean and standard deviation are indicated for each concentration of $\mathrm{Cu}$. Different letters indicate significant differences between different concentrations of $\mathrm{Cu}$. There were no significant differences between populations $(\mathrm{P}>0.05)$. 
las plantas cuentan con una diversidad microbiológica en el suelo, que no se reproduce en los ensayos hidropónicos. Si bien en sitios contaminados es esperable que la diversidad de microorganismos del suelo esté disminuida respecto a un suelo normal (De Val et al., 1999), en estos sitios se ha comprobado que son capaces de sobrevivir ciertos tipos de hongos arbusculares micorrízicos (HAM). Se ha comprobado que ciertos HAM pueden interactuar con plantas en ambientes extremos, mejorando su sobrevivencia (Carlvalho et al., 2006). En la zona de Los Maitenes, Cornejo et al. (2008) estudiaron la presencia de HAM en 4 especies; si bien la presencia de hongos micorrízicos fue escasa en la mayoría de las especies estudiadas (entre 100 y 200 esporas/g), en Oenothera affinis la presencia fue significativamente mayor (600 esporas/g de suelo) predominando el género Glomus. Ensayos posteriores realizados por Meier et al. (2011), adicionando estas micorrizas nativas a suelos contaminados, demostraron que las plantas micorrizadas sobrevivieron a concentraciones de $\mathrm{Cu}$ total de $500 \mathrm{mg}$ $\mathrm{kg}^{-1}$ en comparación a las plantas sin micorrizas o con micorrizas de suelos no contaminados, las cuales no sobrevivieron a estas concentraciones de cobre.

\section{DIFERENCIAS ENTRE LAS POBLACIONES}

Las poblaciones de Oenothera picensis provenientes de Los Maitenes y Maitencillo no mostraron diferencias significativas respecto a las variables de clorosis, producción de biomasa aérea o radical, tampoco sus relaciones $\mathrm{Cu}_{\mathrm{ta}} /$ $\mathrm{Cu}_{\mathrm{r}}$ fueron diferentes (Tabla III). Esto indica que no hubo diferencias en el grado de tolerancia a la concentración de $\mathrm{Cu}$ entre ambas poblaciones, de esta forma el $\mathrm{Cu}$ no es el principal elemento limitante para el crecimiento de las plantas en el sector de Los Maitenes. La aparición de posibles adaptaciones en esta población debe ser evaluada mediante un estudio especializado.

\section{ACUMULACIÓN DE COBRE EN BIOMASA AÉREA}

Las máximas concentraciones de cobre en tejidos aéreos determinadas en esta investigación superaron los 1.000 $\mathrm{mg} \mathrm{kg}^{-1}$ (Fig. 1D). Sin embargo la planta presentó severos síntomas de toxicidad, inhibiéndose en más de un 50\% la producción de biomasa aérea y radical (Fig. 1B y 1C) y causando clorosis en más del $50 \%$ de la superficie foliar (Fig. 1A). Una planta hiperacumuladora es aquella capaz de acumular más de $1.000 \mathrm{mg} \mathrm{kg}^{-1} \mathrm{de} \mathrm{Cu}, \mathrm{Cd}, \mathrm{Cr}, \mathrm{Pb}, \mathrm{Ni}$ o Co, sin mostrar síntomas de toxicidad (Lasat 2000; Whiting et al. 2002). En consecuencia, esto reafirma la conclusión de que Oenothera picensis no se puede considerar una planta tolerante/hiperacumuladora, sino que es más bien una especie sensible.

\section{BIBLIOGRAFÍA}

Ali, N., P. Bernal \& M. Ater. 2002. Tolerance and bioaccumulation of copper on Phragmites australis and Zea mays. Plant and Soil 239(1): 103-111.

Ávila, G., H. Gaete, M. Morales \& A. Neaman. 2007. Reproducción de Eisenia foetida en suelos agrícolas de áreas mineras contaminadas por cobre y arsénico. Pesquisa Agropecuaria Brasileira 42(3): 435-441.

Ávila, G., H. Gaete, S. Sauvé \& A. Neaman. 2009. Organic matter reduces copper toxicity for the earthworm Eisenia fetida in soils from mining areas in central Chile. Chilean Journal of Agricultural Research 69(2): 252-259.

Baker, A. \& R. Brooks. 1989. Terrestrial higher plants which hyperaccumulate metallic elements: A review of their distribution, ecology and phytochemistry. Biorecovery 1(2): 81-108.

Baker, A., W.H. Ernst, A. Van der Ent, F. Malaisse \& R. GinocCHIO. 2010. Metallophytes: the unique biological resource, its ecology and conservational status in Europe, central Africa and Latin America. In: L. Batty \& K. Hallberg (eds.), Ecology of Industrial Pollution, 349 pp. Cambridge University Press, New York, USA.

BaKer, A., S.N. Whiting \& D. Richards. 2002. Metallophytes: a unique biodiversity resource "owned" by the mining industry. $9^{\text {th }}$ New Phytologist Symposium Heavy Metal and Plants. University of Pensylvania, Philadelphia, Pensylvania, USA.

Borghi, M., R. Tongnetti, G. Monteforti \& L. Sebastiani. 2007. Responses of Populus x euramericana ( $P$. deltoides x $P$. nigra) clone Adda to increasing copper concentration. Environmental and Experimental Botany 61(1): 66-73.

Bruno, C. 1992. El viento y el relieve como factores geográficos determinantes en la dispersión de contaminantes derivados de los procesos productivos del cobre (comuna de Puchuncaví, V Región). Tesis. Magíster en Asentamientos Humanos y Medio Ambiente. Facultad de Arquitectura, Diseño y Estudios Urbanos, Universidad Católica de Chile. Santiago, Chile. 103 pp.

Carvalho, L., I. CaÇador \& M. Martinis-Loução. 2006. Arbuscular mycorrhizal fungi enhance root cadmium and copper accumulation in the roots of the salt marsh plant Aster tripolium. Plant and Soil 285(1-2): 161-169.

Chipeng, F., C. Hermans, G. Colinet, M.-P. Faucon, M. Ngongo, P. Meerts \& N. Verbruggen. 2010. Copper tolerance in the cuprophyte Haumaniastrum katangense (S. Moore) P.A. Duvign. \& Plancke. Plant and Soil 328(1-2): 235-244.

Cosio, F., M. Negrón, J. Gastó \& G. Villate. 2007. Distritos y Sitios de la Provincia Templada Secoestival Nubosa o Valparaíso: secano de la costa. Revista de la Ciencia del Suelo y Nutrición Vegetal 7(1): 38-61.

De Gregori, I., G. Lobos, S. Lobos, H. Pinochet, M. PotinGautier \& M. Astruc. 2000. Copper and selenium in rainwater, soils and alfalfa from agricultural ecosystems of Valparaíso Region, Chile. Boletín de la Sociedad Chilena de Química 45(1): 131-146.

De Val, C., J.M. Barea \& C. Azcón-Agullar. 1999. Diversity of arbuscular mycorrhizal fungus population in heavy metal contaminated soils. Applied and Environmental Microbiology 65(2): 718-723. 
Ebbs, S. \& L.V. Kochian. 1998. Phytoextraction of zinc by oat (Avena sativa), barley (Hordeum vulgare), and Indian mustard (Brassica juncea). Environmental Science \& Technology 32(6): 802-806.

Folchi, M.A. 2006. Historia ambiental de las labores de beneficio en la minería del cobre en Chile, siglos XIX y XX. Tesis. Doctorado en Historia Económica. Departamento de Economía y de Historia Económica, Universitat Autónoma de Barcelona. Barcelona, España. 727 pp.

Ginocchio, R. 1997. Aplicabilidad de los modelos de distribución espacio-temporales de la vegetación en ecosistemas sujetos a procesos de contaminación. Tesis. Doctor en Ciencias Biológicas, mención Ecología. Departamento de Ecología, Pontificia Universidad Católica de Chile. Santiago, Chile. $209 \mathrm{pp}$.

Ginocchio, R. 2000. Effects of a copper smelter on a grassland community in the Puchuncaví Valley, Chile. Chemosphere $41(1-2): 15-23$.

Ginocchio, R., G. Carvallo, I. Toro, E. Bustamante, Y. Silva \& N. Sepúlveda. 2004. Micro-spatial variation of soil metal pollution and plant recruitment near a copper smelter in central Chile. Environmental Pollution 127(3): 343-352.

Giri, A.K. \& R.K. PAtel. 2012. Phytoaccumulation potential and toxicity of arsenic ions by Eichhornia crassipes in hydroponic systems. Bioremediation \& Biodegradation 3: 137 doi:10.4172/2155-6199.1000137.

Goecke, P., R. Ginocchio, M. Mench \& A. Neaman. 2011. Amendments promote the development of Lolium perenne in soils affected by historical copper smelting operation. International Journal of Phytoremediation 13(6): 552-566.

GonzÁlez, S. \& E. Berqvist. 1986a. El impacto de emisiones de gases y otros productos desde chimeneas de fundiciones de minerales sobre las actividades agropecuarias. Ambiente y Desarrollo 2(3): 117-122.

GonzÁlez, S. \& E. Berqvist. 1986b. Evidencias de contaminación con metales pesados en un sector del secano costero de la V Región. Agricultura Técnica 46(3): 299-306.

González, S. \& R. Ite. 1992. Acumulación metálica en suelos del área bajo influencia de las chimeneas industriales de Ventanas (Provincia de Valparaíso, V Región). Agricultura Técnica 50(2): 214-219.

González, I., V. Muena, M. Cisternas \& A. Neaman. 2008. Acumulación de cobre en una comunidad vegetal afectada por contaminación minera en el valle de Puchuncaví, Chile central. Revista Chilena de Historia Natural 81(2): 279291.

Gustafsson, J. 2011. Visual MINTEQ VERSION 3.0. Department of Land and Water Resources Engineering, Kth Royal Institute of Technology. Estocolmo, Suecia. URL: http:// vminteq.lwr.kth.se/. Viewed: 2 de junio 2015.

Kopittke, P., F. Blamey, C. Asher \& N. Menzies. 2010. Trace metal phytotoxicity in solution culture: A review. Journal of Experimental Botany 61(4): 945-954.

Kuzovkina, Y., M. Knee \& M. Quigley. 2004. Cadmium and copper uptake and translocation in five willow (Salix L.) species. International Journal of Phytoremediation 6(3): 269-287.

Lasat, M. 2000. The Use of Plants for the Removal of Toxic Metals from Contaminated Soil. Environmental Protection Agency, Washington. 33 pp.
Lou, L.-Q., Z.-G. Shen \& X.-D. Li. 2004. The copper tolerance mechanisms of Elsholtzia haichowensis, a plant from copper-enriched soils. Environmental and Experimental Botany 51(2): 111-120.

Macnair, M.R., G.H. Tilstone \& S. E. Smith. 1993. The genetic of metal tolerance and accumulation in vascular plants. New Phytologist 124(4): 541-559.

Matthei, O. 1995. Manual de las malezas que crecen en Chile. Alfabeta Impresores, Santiago, Chile. 537 pp.

Meier, S., R. Azcón, P. Cartes, F. Borie \& P. Cornejo. 2011. Alleviation of $\mathrm{Cu}$ toxicity in Oenothera picensis by copper-adapted arbuscular mycorrhizal fungi and treated agrowaste residue. Applied Soil Ecology 48(2): 117-124.

Minitab Inc. (2009). Minitab 15. Pensylvania, USA. Disponible: www.minitab.com.

Muena, V., I. GonzÁlez \& A. Neaman. 2010. Efectos del encalado y la fertilización nitrogenada sobre el desarrollo de Oenothera affinis en un suelo afectado por la minería del cobre. Journal of Soil Science and Plant Nutrition 10(2): 102-114.

Quian, M., X. Li \& Z. Shen. 2005. Adaptative copper tolerance in Elsholtzia haichowensis involves production of $\mathrm{Cu}-$ induced thiol peptides. Plant Growth Regulation 47(1): $65-73$.

Rachou, J., C. Gagnon \& S. Sauvé. 2007. Use of an ion selective electrode for free copper measurements in low salinity and low ionic strength matrices. Environmental Chemistry 4(2): 90-97.

SAdZAWKA, A. 2006. Métodos de análisis de aguas para riego. Instituto de Investigaciones Agropecuarias, Santiago, Chile. 332 pp.

Sadzawka, A., M.A. Carrasco, R. Demanet, H. Flores, R. Grez, M.L. Mora \& A. Neaman. 2007. Métodos de análisis de tejidos vegetales. Instituto de Investigaciones Agropecuarias, Santiago, Chile. 139 pp.

Sauvé, S., M.B. Mc Bride, W.A. Norvell \& W. Hendershot. 1997. Copper solubility and speciation of in situ contaminated soils: Effects of copper level, $\mathrm{pH}$ and organic matter. Water, Air and Soil Pollution 100 (1-2): 133-149.

Schiavon, M., L. Zhang, S. Abdel-Ghany, M. Pilon, M. Malagoli \& E. Pilon-Smiths. 2007. Variation in copper tolerance in Arabidopsis thaliana accession Columbia, Landsberg erecta and Wassilewskija. Physiología Plantarum 129: 342-350.

Secretaría Ministerial de Vivienda y Urbanismo de la Región de Valparaíso. 2004. Diagnóstico Ambiental a la Modificación al Plan Regulador Intercomunal de Valparaíso en el Área Metropolitana de Valparaíso y Satélite Borde Costero Quintero-Puchuncaví. Corporación Chile Ambiente, Chile. 162 pp.

Secretaría Regional de Agricultura Región de Valparaíso. 2013. Situación hídrica actual de la Región de Valparaíso. Presentación pública. Quillota, Chile. URL: http://valparaiso.minagri.gob.cl/wp-content/uploads/ sites/7/2013/10/2_SEREMI-de-Agricultura_SituacionHidrica-Actual-de-la-Region-de-Valparaiso_13-5-13.pdf. Visto: 31 de mayo de 2015.

Soll Survey Staff. 2003. Keys to soil taxonomy. United States Department of Agriculture. National Resourses Conservation Service, Washington, DC, USA. 332 pp. 
Steubing, L. 1982. Problems of bioindication and the necessity of standardization. In: L. Steubing \& H. Jäger (eds.), Monitoring Air Pollutants by Plants, pp. 19-27. Dr. Junk Publishers, The Hague, The Netherlands.

Stuckey, J., A. Neaman, R. Ravella, S. Komarnei \& C. Martínez. 2009. Highly charged swelling mica reduces $\mathrm{Cu}$ bioavailability in $\mathrm{Cu}$-contaminated soils. Environmental Pollution 157(1): 12-16.

VERLINDEN, M. 1982. On the acid decomposition of human blood and plasma for the determination of selenium. Talanta 29(10): 875-882.

Wenshan, K., Z.-T. Xiong, C. Shijan \& C. Jianjung. 2007. Effects of copper and mineral nutrition on growth, copper accumulation and mineral element uptake in two Rumex japonicus population from a copper mine and an uncontaminated field sites. Environmental and Experimenal Botany 59(1): 59-67.

Whiting, S.N., R.D. Reeves \& A.J.M. BaKer. 2002. Conserving biodiversity: Mining metallophytes and land reclamation. Mining Environmental Management 10(2): 11-16.

Whiting, S.N., R.D. Reeves, D. Richards, M.S. Johnson, J.A. Cooke, F. Malaisse, A. Paton, J.A.C. Smith, J.S. Angle, R.L. Chaney, R. Ginocchio, T. Jaffré, R. Johns, T. McIntyre, O.W. Purvis, D.E. Salt, H. Schat, F.J. Zhao \& A.J.M. BAKER. 2004. Research priorities for conservation of metallophyte biodiversity and their potential for restoration and site remediation. Society for Ecological Restoration International 12(1): 106-116.

Recibido: 06.01.15

Aceptado: 27.08.15 